\title{
Bemerkungen über den Islam in Nordabessinien.
}

\author{
Von \\ Enno Littmann.
}

Es ist eine aus Reisewerken und Missionsberichten längst bekannte Tatsache, daß der Islam in Nordabessinien während des I9. Jahrhunderts große Fortschritte gemacht hat. Ganze Stämme, die sich einst zum abessinischen Christentum bekannt haben und noch christliche Namen tragen, sind Mohammedaner geworden. Man denke nur an die drei mohammedanischen Stämme, die sich Taklës "Pflanze Jesu" (für Takla 'Iyasüs), Hebtès "Gabe Jesu» (für Hebta 'Iyasūs) und Temäryām "Gabe Mariä» (für Hebta Märyäm) benennen! Wann und unter welchen Bedingungen diese und andere Stämme zum Islam übergetreten sind, darüber gibt uns meist nur die einheimische Überlieferung Aufschluß. Werner MunzInger hat in seinem außerordentlich anregenden und inhaltsreichen Buche "Ostafrikanische Studien" (Schaffhausen, 1864) als einer der Ersten diese Quellen erschlossen. Mancherlei Material über diese Frage findet sich auch in den Büchern und Aufsätzen von italienischen Gelehrten und Reisenden, die das Leben der Völkerschaften ihrer Colonia Eritrea' studiert haben ${ }^{\mathrm{I}}$ ). Sehr verdienstlich haben sich dann die schwedischen Missionare in Gäläb (zwischen Massaua und Kärän) gemacht durch Aufzeichnung der Überlieferungen der Tigrē-Stämme. Einiges habe ich selbst während meines Aufenthaltes im Lande beobachten und aufzeichnen können. Eine Einzeluntersuchung, die all das zerstreute Material berücksichtigt, würde vielleicht allerhand interessante Aufschlüsse bringen.

Hier möchte ich nur kurz zusammenstellen, welche Fortschritte der Islam in jenen Gegenden unter den früher christlichen Stämmen während der letzten 50 Jahre gemacht hat. Dazu dienen mir einerseits die Tabellen Munzinger's (a. a. O. S. 79-8I), die die Verhältnisse um 1860 wiedergeben, und andererseits meine eigenen Aufzeichnungen,

) Vgl. besonders Contr Rossinr, Il Censimento delle Popolazioni indigene della Colonia Eritrea, Firenze, 1902. 
durch die der Stand der Dinge um 1905 geschildert wird. Sie bilden den nächsten sicheren Ausgangspunkt, und ein Vergleich der beiden ist recht instruktiv.

Gehen wir von. Massaua aus nach Nordwesten, so treffen wir von den hier in Frage kommenden Stämmen zunächst die beiden Mensa $\left(M a ̈ n s a^{*}\right)$. Nördlich von ihnen zelten die drei größten TigrēStämme, die "Drei Maflas" (oder "Drei H̦abāb»), d. i. die "Ad Temāryām, die 'Ad Taklēs und die 'Ad Hebtēs, die jedoch meist die Habāb par excellence genannt werden. Westlich von den Mänsa' wohnen die Bogos, Bēt Gúuk (bei Munzinger und den Italienern Bedjuk) und die Bēt 'Auque (bei Munzinger und den Italienern Beit Takue). Nordwestlich von den Mänsa' endlich finden wir die beiden Mārya-(Marea-) Stämmẹe, d. i. die Schwarzen Mārya und die Roten Mārya.

Von allen diesen Stämmen sind die $\mathrm{Mäns} \mathrm{a}^{\circ}$ und ihr TigrēDialekt am besten bekannt. Sie zerfallen in die beiden Zweige der Bēt-Abrěhe $\bar{e}^{x}$ ) und Bēt-Šahaqan ${ }^{2}$ ); letztere wohnen südlich von den ersteren. Sie sind beide Halbnomaden, bringen den Sommer im Hochlande, den Winter im Tieflande zu. MunzINGER bezeichnet sie sämtlich als Christen. Mir wurde 1905/6 über die Mänsa' berichtet: - Ihre Religion war früher das .Christentum und beide (Zweige) hatten eine Kirche und Priester. Aber später verstanden ihre Priester nicht mehr zu lesen. Dann kamen die Muslime in ihr Land und bekehrten sie zum Islam. Und die meisten von ihnen wurden Muslime; nur einige wenige leben noch bis auf den heutigen Tag als Christen. "Etwa $2 / 3$ der Mänsa' sind heute mohammedanisch, $1 / 3$ ist noch christlich. Die schwedische Mission in Gäläb jedoch hat dem Vordringen des Islam mit Erfolg Widerstand geleistet, auch in manchen Fällen Mohammedaner wieder zum Christentum zurückgeführt, da hier in Nordabessinien der Abfall vom Islam durchaus nicht so scharf beurteilt wird wie z. B. in Arabien, Syrien und Persien.

Von den $\triangle \mathrm{D}$ rei $\mathrm{H}$ a bāb* sagt Munzinger, sie seien seit 40 Jahren Mohammedaner. $\mathrm{DaB}$ sie früher Christen gewesen sind, beweisen nicht nur die Namen, sondern auch Kirchenruinen in ihrem Lande, wie z. B. in Hagara Năgrām. Dazu kommen noch manche christliche Formeln und Gebräuche, die sich bei ihnen erhalten haben; vor allem aber die Uberlieferung selbst. Nach ihr hat vor etwa Io0 Jahren Kantēbãy (d. i. Häuptling) Gáweǵ von den Habăb den Islam angenommen, in dem Glauben, der Islam bringe Glück und langes Leben. Damals sagte er zu seinem Priester: "Zerschlage das

3) Gebort zu 'Abreham (Abraham).

2) Ableitung von' $E ; b a q$ (Isazk). 
Täbot (" I) Dẹr Priester aber sagte: „Ich wage nicht, das Täböt Mariae. zu zerschlagen.. Und Kantēbðy Gảweǵ nahm das Täböt mit eigenen. Händen und zerhieb es mit einer Axt. Dann nahmen auch die Priester den Islam an; und alle ihre Nachkommen sind die Schēche des Stammes bis auf den heutigen Tag. - Das Verwandtschaftsverhältnis der drei Stämme wird am besten durch folgenden Stammbaum dargestellt:

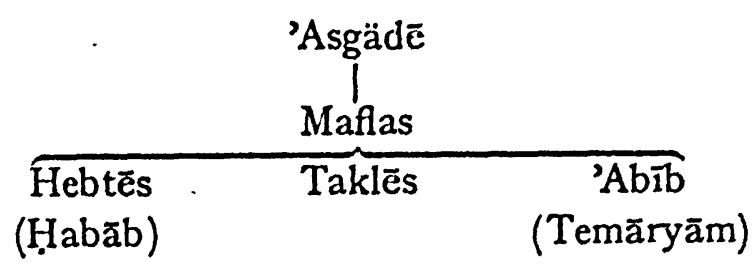

Die Bogos (im Tigrē häufig nach ihrer Sprache Belēn genannt) waren zu MunziNGER's Zeit "Christen, wenige Mohammedaner". Mir wurde von ihnen erzählt: "Sie waren einst alle christlich; jetzt aber ist die Hälfte von ihnen mohammedanisch». Auch hier können wir ein starkes Anwachsen des Islam konstatieren.

Die Bēt Gūk (Bedjuk) werden von Munzinger als pparitätisch» bezeichnet. Jetzt sind sie alle mohammedanisch; aber sie berichten noch, daB sie einst Christen waren.

Die $B \bar{e} t$ 'A u q e (Beit Takue). waren schon zu Munzinger's Zeit seit 20 Jahren mohammedanisch. Die Erinnerung an ihr früheres Christentum ist ihnen natürlich geblieben, ebenso wie den beiden Mārya, die um 1860 "seit 40 Jahren" islamisch waren.

In dieser Übersicht sind unberücksichtigt geblieben I. diejenigen christlichen Gegenden Nordabessiniens, in denen der Islam schon vor I800 Eingang gefunden hat; 2. die heidnischen Stämme, die vor oder nach I800 islamisch geworden sind; 3. die arabischen Stämme, die von der ànderen Seite des Roten. Meeres eingewandert sind.

- Die Fortschritte, die der Islam unter den früher christlichen Tigrē-Stämmen Nordabessiniens im I9. Jahrhundert gemacht hat, lassen sich also tabellarisch etwa folgendermaßen darstellen:

\begin{tabular}{|c|c|c|}
\hline - & Religion um 1860 & Religion um 1905 \\
\hline 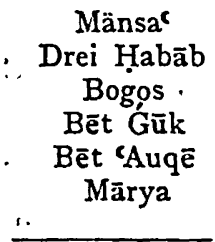 & $\begin{array}{l}\text { Christen } \\
\text { Seit } 40 \text { Jahren Mohammedaner } \\
\text { Christen, wenige Mohammedaner } \\
1 / 2 \text { Christen, } 1 / 2 \cdot \text { Mohammedaner } \\
\text { Seit } 20 \text { Jahren Mohammedaner } \\
\text { Seit } 40 \text { Jahren Mohammedaner }\end{array}$ & $\begin{array}{c}\text { 2/3 Mohammedaner, } 1 / 3 \text { Christen } \\
\text { 1/2 Mohammedaner, } \pi / 2 \text { Christen } \\
\text { Mohammedaner } \\
\text {. . . - }\end{array}$ \\
\hline
\end{tabular}

1) Tābōt ist ursprünglich die "Bundeslade«, die in Aksum aufbewahrt wird. Dann wird auch jede Lade oder verzierte Holzplatte, die vom Priester zum Aufenthaltsorte der Gottheit geweiht ist, so bezeichnet. 
Eine kurze Übersicht über die' Stämme Nordabessiniens, die Tigre sprechen oder es verstehen, und über ihre Religion und Lebensweise findet man in meinen Publications of the Princeton Expedition to Abyssinia, Leiden I9IO, Bd. II, S. 335-344. In demselben Bande findet sich auch noch anderes hierher gehörige Material an verschiedenen Stellen. Hier sei es mir gestattet, noch auf zwei besonders instruktive Fälle von religiösem Synkretismus aus jenen Gegenden hinzuweisen.

Mein früherer Diener Naffa' wad 'Etmān erzählte mir, er habe einst - vor etwa 8 Jahren - auf dem Wege nach Agordat (Aqerdät) einen muslimischen Amharer getroffen, der sich als Prophet ausgab. Der Mann berichtete von allen möglichen Wundern und Zeichen, die er im Namen Allah's getan habe, die Naffa und sein Gefährte Pẹtrōos aus Gäläb ihm aber nicht glauben wollten. Unter anderem sagte er auch, er habe auf Löwen geritten ${ }^{1}$ ). Dieser selbe "Prophet" pflegte, wenn er'seine Liturgie sang, die christliche Ge'ez-Liturgie zu singen, soweit er sie in Erinnerung hatte. Als Naffa' ihn darauf aufmerksam machte, behauptete er, die Liturgie sei muslimisch!

Ferner hat mir Naffa' folgendes aufgeschrieben, das ich wörtlich übersetze: Einige Leute redeten über Religion. Mekkēēl wad Gärgīs (d. i. Michael, Sohn des Georgios) aus Dängäreǵba (südwestlich von Gälăb) sagte: „Obgleich ich ein Christ bin, pflegte ich doch auch fest an die Schēche zu glauben, (nämlich) daß sie einige Wunder tun könnten. Aber einmal kam ein berühmter Schēch in unser Dorf. Und ich nahm ihn gut auf, damit ich durch ihn Segen erhielte. Als er dann aufbrechen wollte, beschloB ich mit meiner Frau, ihm etwas Geld zu geben. Und wie er aufbrach, sah er meine Mauleselin und sprach zu mir: :Du hast Glück; jetzt wird auch diese deine Mauleselin ein weibliches Füllen 2) zur Welt bringen ! « Denn da er sah, daß die Mauleselin feist war, glaubte er, sie sei trächtig. Danach habe ich den Schēchen keinen Glauben mehr geschenkt; das war zu viel für meinen Glauben!*3)

3) Von den christlichen Heiligen in Abessinien wird ebenso wie von den muslimischen in Kleinasien und den buddhistischen in Tibet erzählt, daB sie auf Löwen reiten; vgl. u. a. JACOB, Die Beklaschijie in ihrem Verhälnis zu verwandlen Erscheinungen, S. 8.

2) Weibliche Junge von Haustieren werden als besonderer Segen angeschen.

3) Die Maultiere sind bekanntlich unfähig, sich fortzupflanzen. 